\title{
A DNA delivery system targeting dendritic cells for use in immunization against malaria: a rodent model
}

\author{
G.B. KETO ${ }^{\prime *}$, S.E. WEST ${ }^{2}$, S. STEIGER ${ }^{3}$, H-P. BECK ${ }^{3}$, N. WEISS ${ }^{3}$ \& N.E. HYNES ${ }^{4}$ \\ ${ }^{\prime}$ National Institute for Medical Research, Tanga Research Centre, P.O. Box 950, Tanga, Tanzania \\ ${ }^{2}$ Department of Pathology \& Microbiology, University of Bristol, Bristol, UK \\ ${ }^{3}$ Swiss Tropical Institute, Basel, Switzerland \\ ${ }^{4}$ Friedrich Miescher Institute, Basel, Switzerland
}

\begin{abstract}
DNA-based vaccination has emerged as a promising method of immunisation since the first demonstration of this technology. Improving the antibody responses is desirable for the protective efficacy and hence broad application of these vaccines. We examined the immunogenicity of a Plasmodium-based DNA vaccine that was targeted to antigen presenting cells by fusion to CTLA4. Fusion proteins comprising the extra-cellular domain of CTLA4, the hinge, CH2 and $\mathrm{CH} 3$ domains of human IgG1 and MSP-1 gene fragments were expressed in COS-7 cells. Three of the secreted proteins containing the mouse homologue of CTLA4 were shown to bind differently to the human B7-1 molecule expressed on THP-1 cells. Competition binding assays for two fusion proteins showed that binding was specific. When C57BL/6 mice were immunized with plasmids encoding the fusion proteins, antibodies against two denatured and one non-denatured MSP-1 gene fragments were successfully induced. The usefulness of this strategy in future studies of immunisaton against human malaria is discussed.
\end{abstract}

Key words: malaria, PbMSP-1, DNA vaccine, dendritic cells, rodent model

\section{Introduction}

Malaria remains the most prevalent devastating parasitic disease worldwide. Among the four different Plasmodium species that cause malaria in humans, the greatest mortality and morbidity is associated with $P$. falciparum. With the widespread development of drug resistance in the parasite, vaccination is considered to be an approach that will complement other strategies for prevention and control of the disease in future. It has become well established that protective immune responses to the parasite are directed at stage-specific antigens. Proteins expressed at different stages of the life cycle of $P$. falciparum are likely to be involved in the generation of natural immunity to malaria and a number of these proteins have been highlighted as candidates for a subunit vaccine (Soares et al., 1997). Merozoites, which are the extracellular developmental form of the parasite that invade erythrocytes, expose on their surface a protein complex, which is the processed product of a 190$200 \mathrm{kDa}$ precursor known as merozoite surface protein-1 (MSP-1). MSP-1 was first described in $P$. yoelii (PyMSP-1) as a protein that elicited a protective immune response in mice (Aikawa et al., 1977). It has been subsequently identified and characterized in many other Plasmodium species. However expression of full-length recombinant PfMSP-1 in heterologous systems has turned out to be most difficult if not impossible (Sandhu \& Kennedy, 1994) due to the high AT content of $P$. falciparum DNA that prevents the cloning and stable maintenance of large genes in Escherichia coli.

The $19 \mathrm{kD}$ C-terminal part of PfMSP-1 is a leading malaria vaccine candidate and has been shown to protect against malaria in several studies (Hirunpetcharat et al., 1997). Studies with monoclonal antibodies and the cloning of the MSP1 from a number of parasite isolates revealed that this antigen is polymorphic (McBride et al., 1985). In human malaria, detailed sequence analysis of four cloned genes suggests that the gene consists of only two prototype sequences (Tanabe et al., 1987). Although the correlates for immune protection against malaria infection are currently unknown, it is generally agreed that an effective malaria vaccine should elicit both strong neutralizing antibody and cytotoxic T lymphocyte (CTL) responses (Hoffman et al., 1994).

Vaccines that mimic the antigenicity of infectious organisms may ultimately prove to be the most effective strategy for achieving protective

* Correspondence: G.B. Keto: Email: mechtilda@yahoo.com 
immune responses. In this respect, a DNA vaccine has significant advantages over alternative immunization strategies, and represents a novel means of expressing antigens in vivo for the generation of protective immune responses. Intramuscular or intradermal injection of antigen encoding plasmid DNA induces both cellular and humoral immune responses and holds great vaccination potential (Sedegah et al., 1994). The results from a study comparing the humoral immune response elicited by DNA and protein vaccination showed that the protein immunized mice resolved their parasitemia whereas the DNA-immunized mice died of infection. This was mainly attributed to the production of antibodies, which had significantly lower titre, avidity and different isotype profile in the DNA-immunized mice. Other studies have shown that DNA vaccines against malaria are partially protective (Gardner et al., 1996). In a non-human primate model, the results from a multistage $P$. falciparum DNA vaccine, NYVAC-PF7, have shown that the vaccine was safe and tolerated but had variable immunogenicity (Ockenhouse et al., 1998).

Expression of genes in recombinant organisms is important to produce protein for the development of vaccines against pathogens. For eukaryotic pathogens or viruses it is often necessary to express the proteins in a conformation that closely resembles that of the native protein, with the appropriate formation of disulfide bonds and modifications such as $\mathrm{N}$-glycosylation. Attempts to produce a secreted form of the native cysteine rich, carboxyl-terminal $42 \mathrm{kD}$ processing fragment of PfMSP-1 in yeast were not successful (Hirunpetcharat et al., 1997).

Here we describe the expression and characterization of site-directed recombinant $P$. berghei MSP-1 (PbMSP-1) fragments that can be utilized, using the primate homologues, in future studies involving the prime-boost strategy. Furthermore, the plasmids encoding the antigens can be used in studies investigating the responses of a DNA vaccine encoding an antigen that is directed to sites of immune induction.

\section{Materials and Methods}

\section{Cells lines and cell culture conditions}

The acute monocytic leukaemia cell line THP-1 (ATCC) and B7-transfected THP-1 cells (kindly provided by A. Lanzavecchia, Basel) were maintained in RPMI 1640 medium supplemented with $2 \mathrm{mM}$ glutamine, $50 \mu \mathrm{M}$ ß-mecaptoethanol, 5 $\mathrm{mM}$ Sodium pyruvate, $5 \mathrm{mM}$ non-essential amino acids, $10 \%$ heat inactivated fetal calf serum (FCS), and $1 \mathrm{mg} / \mathrm{ml} \mathrm{G} 418$ (THP-1-B7-1). COS7 cells were cultured at $37^{\circ} \mathrm{C}$ in Dulbecco's modified eagle's medium, DMEM, supplemented with $10 \%$ FCS and a humidified environment containing $5 \% \mathrm{CO}_{2}$. Bacteria were grown and transfected according to established protocols.

Table 1: Ligonucleotides used in plasmid constructs for A) mammalian and B) bacterial expression

A)

\begin{tabular}{|ccll|}
\hline fragment & size $(\mathrm{bp})$ & \multicolumn{1}{c|}{ Sense primer } & \multicolumn{1}{c|}{ Antisense primer } \\
\hline A & 733 & ccaatgcatGCATCACCACCAAGTG & cgggatccTTAAGCGGGTTCTGTACTAG \\
B & 988 & ccaatgcatAACGAAGCAGATTGC & cgggatccTTAAGCTGCTTCTTTG \\
C & 1018 & ccaatgcatATGGTATTGGTTATC & cggatccTTACGCTGCTGTTGT \\
D & 1331 & ccaatgcatGAAGTTGACAGACTTGCC & cgggatccTTACTCTGATAATTCCTTAAGTGTGCG \\
E & 1051 & ccaatgcatGCCTCATCAACACCAGCAGC & cgggatccTTAACCCGATCCTGATCCCGATTCTGG \\
F & 708 & ccaatgcatGCTTCAGCTTCACCTGCTGCAGC & cgggatccTTAAAGATCTTCAAAGATTGGAAC \\
G & 656 & caactgcagGCTGCTACTACCGATAAAG & cgggatccTTATCCCATAAAGCTGGAAG \\
\hline
\end{tabular}

B)

\begin{tabular}{|ccll|}
\hline fragment & size $(\mathrm{bp})$ & \multicolumn{1}{c|}{ Sense primer } & \multicolumn{1}{c|}{ Antisense primer } \\
\hline A & 733 & gctctagatGCATCACCACCAAGTG & gggcccTTAAGCGGGTTCTGTACTAG \\
D & 1331 & ggtctagaaGTTGACAGACTTGCC & gggcccTTACTCTGATAATTCCTTTAAGTGTGCG \\
G & 656 & ggtctagaaGCTGCTACTACCG & gggcccTTATCCCATAAAGCTGGAAGAGC \\
\hline
\end{tabular}




\section{Plasmid constructs}

The plasmid vector pmoCIg containing the extracellular domain of murine cytotoxic $\mathrm{T}$ lymphocyte antigen CTLA4 (CD 152) (with a native signal sequence), was cloned from plasmid CTLA4.hIgG1.pRep7 (kindly provided by J.S. Boyle, Melbourne, Australia). Total RNA was isolated from $P$. berghei-infected (ANKA strain) red blood cells (RBC) cells by TriZol extraction (Life Technologies Inc., Grand Island, NY) and reverse transcribed using the first strand cDNA synthesis kit (Pharmacia Biotech) according to the manufacturer's instructions. Primers (Table 1A) designed on the basis of the DNA sequence of $P$. berghei (ANKA strain) (Accession number AF187232) were used to amplify MSP-1 gene fragments by the polymerase chain reaction (PCR). The PCR cycle conditions were: initial denaturation $94^{\circ} \mathrm{C}$ for $2 \mathrm{~min}$ followed by 25 cycles of denaturation at $94^{\circ} \mathrm{C}, 1 \mathrm{~min}$; annealing at $56^{\circ} \mathrm{C}, 30$ sec.; extension at $68^{\circ} \mathrm{C}, 30 \mathrm{sec}$. and a final extension cycle at $68^{\circ} \mathrm{C}$ for $10 \mathrm{~min}$. The fragments obtained were used to construct plasmids herein referred to as pmoCIg-X and their protein products as moCIg-X, where $\mathrm{X}$ refers to the specific MSP-1 fragment (Figure 1B).

Plasmids designated phuCIg-X and expressing the extracellular domain of human CTLA4, were cloned from pPIC9-CTLA4 125 (kindly provided by W. Wels, Frankfurt) and pWW15-5R (Beerli et al., 1994).

Bacterial expression plasmids were constructed after amplification of mammalian expression plasmids using primers listed in Table $1 \mathrm{~B}$ and cloning into the pQE31 plasmid (Qiagen). The resulting plasmid constructs are herein referred to as pQM-A, pQM-D, and pQM-G and their protein products as A, D, and G, respectively. E. coli M15 [pREP4] were used for expressing histidine-tagged MSP-1 recombinant proteins according to the manufacturers recommendation and established protocols. Mammalian protein expression was evaluated in vitro by transiently transfecting COS-7 cells using Fugene ${ }^{\circledR} 6$ (Boehringer Mannheim) transfection reagent following the instructions from the manufacturer.

\section{Binding assay}

The binding of soluble fusion proteins to B7-1 was evaluated by flow cytometry using a single cell suspension of $5 \times 10^{5}$ THP-1-B7-1 cells and $0.2 \mu \mathrm{g}$ of FITC-conjugated goat anti-human IgG (gamma-

A) Plasmodium berghei MSP1 gene

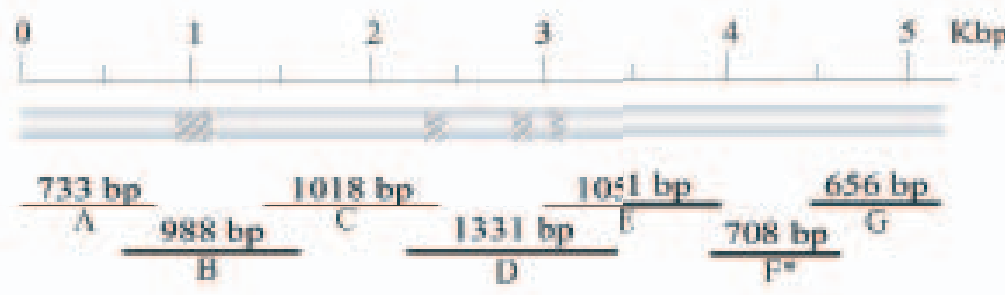

B) Mammalian expression construets

A) moClg-X constructs

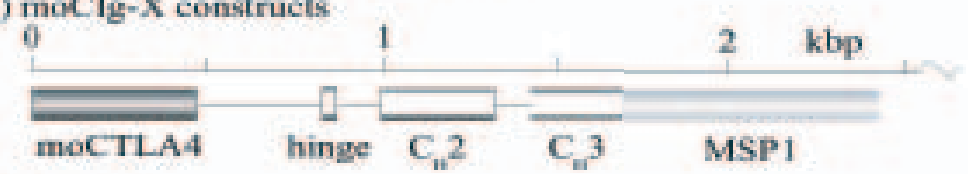

b) huClg-X constructs

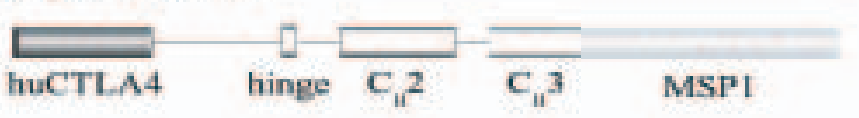

c) huCTLA4 construct

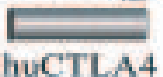

Figure 1: Schematic representation of the $P$. berghei merozoite surface protein 1 gene and expression cassettes chain specific) $\mathrm{F}(\mathrm{ab})_{2}$ antibody (Sigma, Missouri, USA) following established protocols. Parental B71-negative THP-1 cells served as a control. Competitive binding assays were performed using $5 \times 10^{5}$ THP-1B7-1 cells incubated with spent medium containing respective moCIg-X (concentrated 10 fold) proteins with or without huCTLA-4 (at two dilutions having a 10-fold difference).

Immunization of mice with plasmids pmoCIg-X

C57B/6 mice were injected with $100 \mu \mathrm{g}$ of each of the following purified formulations in a total volume of $100 \mu 1$ under the scruff of the neck using a 27 gauge needle; (i) a mixture of plasmids pmoCIg-A, pmoCIg-B and pmoCIg-C 
designated herein as $\mathrm{N}_{\text {mix }}$, (ii) a mixture of plasmids pmoCIg-D, pmoCIg-F, and pmoCIg-G designated as $\mathrm{C}_{\text {mix }}$ (iii) phosphate buffered saline, and (iv) the backbone plasmid, pcDNA 3.1(-). Booster injections were administered at four and eight weeks after initial immunization. Blood was taken before immunization and at week 27 from the tail vein (while under restraint) or periorbital sinus (while the animals were under general anesthesia).

\section{Analysis of sera by Western blotting}

Purified histidine-tagged MSP-1 proteins expressed in bacteria were each run on a single-well 10\% SDSpolyacrylamide gel, electrotransfered onto a nitrocellulose membrane (Millipore Corp., Bedford, $\mathrm{MA}$ ) and incubated for $2 \mathrm{hr}$, at room temperature, with pooled serum (diluted 1:100 in blocking buffer) from mice immunized with either of the formulations described earlier or mouse anti-6XHis antibody (R\&D Systems)(1:1000 dilution). The membrane was developed by incubation with alkaline phosphatase-conjugated goat anti-mouse IgG antibody (Sigma) at 1:2000 dilution.

\section{Results}

Culture media containing secreted fusion proteins was concentrated ten fold and analyzed on a 5$12.5 \%$ SDS polyacrylamide gel. Following transfer to a PVDF membrane, proteins were detected by probing with anti-human or anti-mouse CTLA4 antibodies (Figure 2A and 2B). Both moCIg-F and huCIg-A had an extra band of higher molecular mass. The nature of these extra bands was not investigated further. We were not able to detect moCIg-E using this protocol and no further attempt was made to obtain the fusion protein.

Recombinant histidine-tagged MSP-1 proteins A, D and $\mathrm{G}$, were of the expected sizes as determined by gel electrophoresis and Coommassie blue staining (Figure 2C). Though the same procedure was used for expression and purification, the amount of expressed protein differed between the products.

Fig 2 A.

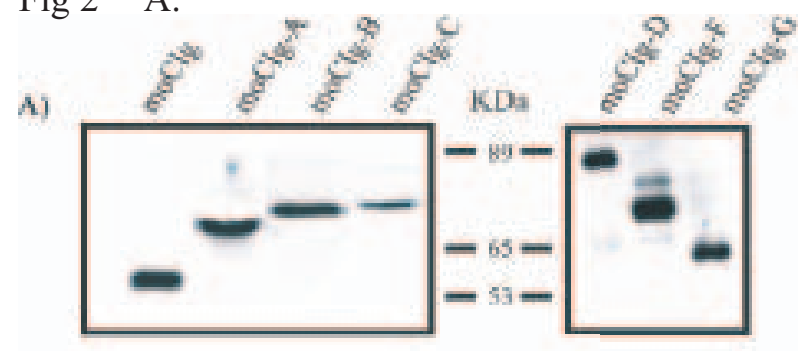

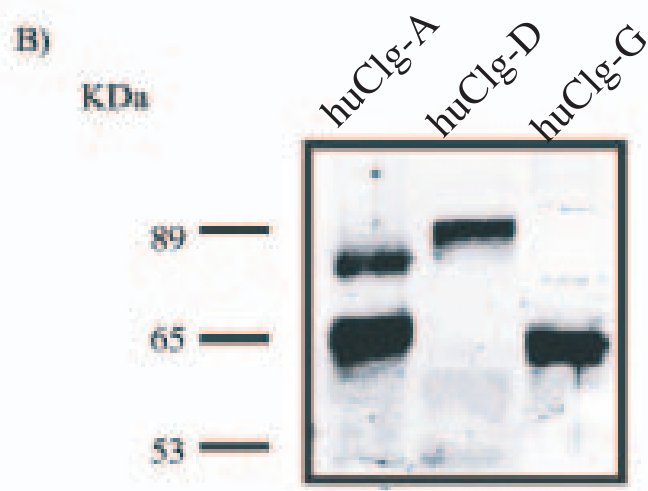

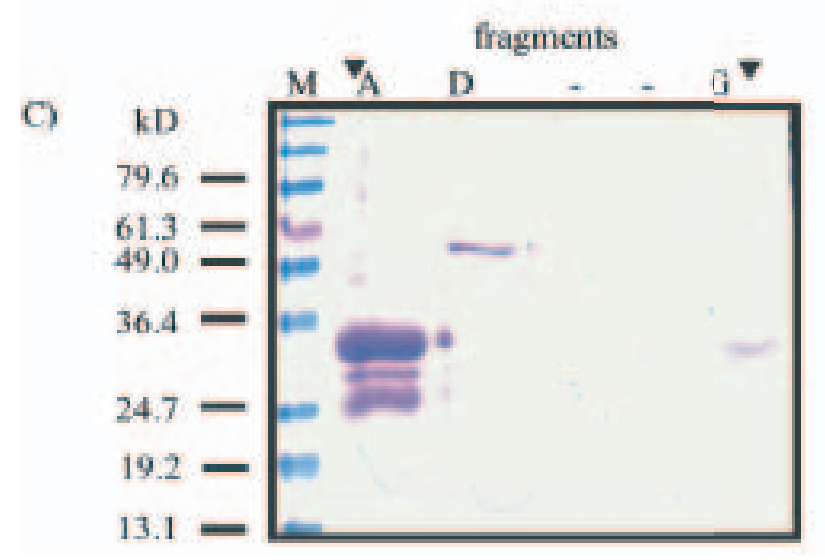

Figure 2: Expression and secretion of moCIg-X, and huCIg-X fusion proteins

To investigate the functional activities of soluble moCIg-X fusion proteins, the binding of the proteins to the $\mathrm{B} 7$ molecule on the surface of cells was tested by flow cytometry. Binding of moCIg-A and moCIg-D to THP-B7-1 but not to B7-negative parental THP-1 cells was detected indicating that the COS-7-expressed fusion proteins were functionally active and could interact with the B7-1 molecule on THP-1-B7-1 cells. On the other hand, moCIg-G showed weak binding to THP-1-B7-1 cells (Figure $3)$. To determine the specificity of interaction between B7-1 and the fusion proteins, a competition binding experiment using the huCTLA4 ${ }_{125}$ fusion protein was attempted. Binding of moCIg-A and moCIg-D fusion proteins was competed in a dose dependent fashion by huCTLA4125. On the other hand, since weak binding was observed for moCIg$\mathrm{G}$, the extent of dose dependency could not be ascertained for this fusion protein (data not shown). 

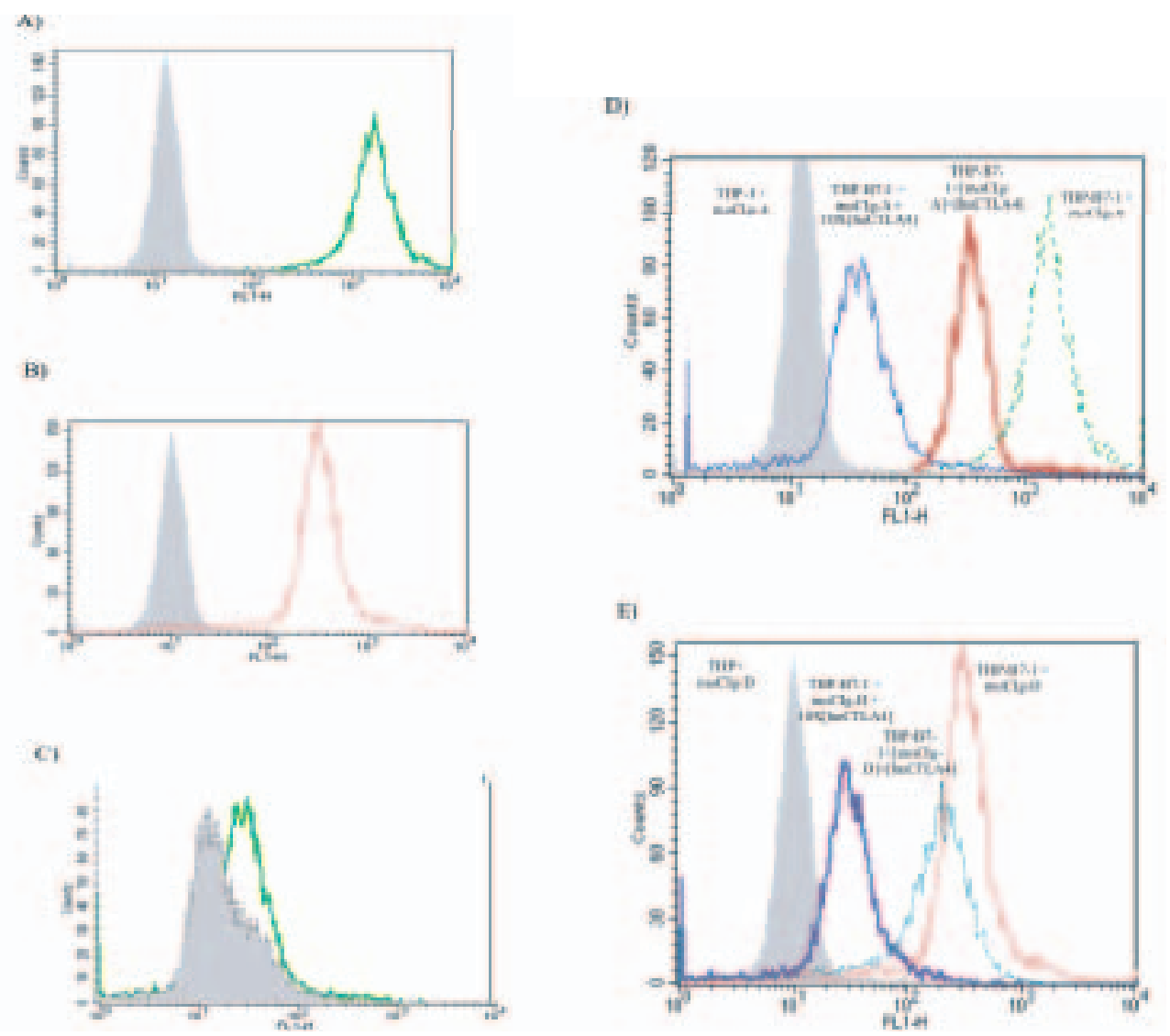

Figure 3: Binding and competing of moCIg-X fusion proteins

To assess the ability of direct injection of the murine constructs in eliciting immune responses, pooled sera from mice were screened for the presence of antibodies. A strong immune response towards fragment A was observed with pooled serum of mice immunized with plasmids from the $\mathrm{N}$-terminal part of MSP-1, $\mathrm{N}_{\text {mix }}$. The immune response towards mice immunized with the C-terminal part of MSP-1, $\mathrm{C}_{\text {mix }}$, was much weaker in detecting fragment $\mathrm{D}$ whereas, reduced fragment $\mathrm{G}$ was not detected at all. Pooled serum from mice immunized with $\mathrm{N}_{\text {mix }}$ was able to weakly recognize fragment $\mathrm{D}$ but not fragment $\mathrm{G}$ and pooled serum from mice immunized with $\mathrm{C}_{\text {mix }}$ was able to weakly detect fragments $\mathrm{A}$.

Since fragment $\mathrm{G}$ contains cysteine residues there was a possibility that disulphide bridges could be formed, therefore the reactivity of pooled serum from mice immunized with $\mathrm{C}_{\text {mix }}$ towards nonreduced fragment $\mathrm{G}$ was investigated. The hyper immune serum recognized the non-reduced form of fragment G. Another prominent band was observed at a size which is approximately twice that expected. None of the sera from immunized mice was able to detect the non-reduced form.

\section{Discussion}

In developing a vaccine that could be used against malaria, we have attempted to produce MSP-1 fusion proteins that are targeted to professional antigen presenting cells. Transient transfection assays were first used to demonstrate that the fusion proteins, incorporating the MSP-1, were synthesized in primate cells. The secreted proteins produced bands of the expected size when subjected to SDSPAGE under reducing conditions and western blotting. The results also show that MSP-1 polypeptide containing the native signal peptide from CTLA4 (in pmoCIg-X) or the IgG signal peptide (in phuCIg-X) could be expressed in COS7 cells. The recognition and processing of signal peptides is essential for the overall efficiency of protein secretion. The heterologous nature of signal peptides used here did not prevent secretion, indicating recognition of the peptides by the secretory machinery of COS 7 cells. Although the general structural features of signal peptides are 
conserved from bacteria to man few malaria parasite gene fragments coding for exported or membrane proteins have been tested for expression in primate cells. More detailed studies are required for establishing whether other malaria parasite signal sequences for endoplasmic reticulum export are recognized in these cells.

Binding studies were undertaken to determine whether the secreted proteins were indeed targeted to the B7-1 molecule. Three fusion proteins were chosen depending on previous documentation of (i) blocking effect, region containing moCIg-A, (ii) presence of B-cell epitopes, region containing moCIg-D, and iii) proven vaccine efficacy, region containing moCIg-G . Binding of moCIg-A, moCIg$\mathrm{D}$ and moCIg-G was demonstrated after incubating THP-1-B7 cells with conditioned medium containing the respective fusion proteins. Efficient binding was observed for moCIg-A and moCIg-D but not for moCIg-G. Competition binding assays performed using huCIg demonstrated that the binding of moCIg-A and moCIg-D to B7-1 was specific. However, the avidity of the binding was not determined.

Bacterial expression constructs of fragments A, $\mathrm{D}$, and $\mathrm{G}$ were made to generate recombinant proteins for later use in specific antibody experiments. Antibodies against reduced fragments $\mathrm{A}$ and $\mathrm{D}$ could be detected even at the 27 -week time point. This indicates that plasmid DNA immunization led to the production of antibodies that were directed against linear epitopes on the proteins. The absence of a reaction with reduced recombinant fragment $\mathrm{G}$ suggests that the antibody titre had diminished to undetectable levels, antibodies generated were directed towards conformational epitopes, or there were no antibodies generated.

The $19 \mathrm{kDa}, \mathrm{C}$-terminal fragment of the major surface protein of $P$. falciparum (PfMSP1 (19)) is a candidate for inclusion in a subunit malaria vaccine. In this study using the rodent model, antibodies were detected which reacted with the non-reduced form of the fragment. The hyperimmune serum also reacted with only this form suggesting that in natural infections, antibodies against this fragment may be directed against conformational epitopes.

The detection of an antibody response against fragment $A$ of mice vaccinated with $C_{\text {mix }}$ seems to indicate that part of fragment $\mathrm{A}$ (from $\mathrm{N}$-terminal portion) contains epitopes that are recognized by antibodies generated by the $\mathrm{C}$ terminal portion of MSP-1. DNA vaccines are considered to have potential advantages because of ease of construction and manipulation, ability to induce long-lasting immune responses, high temperature stability and low production cost. The potency of DNA vaccines has been improved in other systems with the inclusion of immunostimulatory sequences (Sato et al., 1996), DNA-encoding cytokines (Xiang \& Ertl, 1--995), or co-stimulator molecules (Boyle et al., 1999). The results presented here indicate that targeting PbMSP1 gene fragments to APCs results in an antibody response and binding of murine CTLA4 to human B7 shown here may help in elucidating the mechanisms that are different between human and rodent immune induction when doing vaccination studies. Taking into account that very few adjuvants have been approved for use in humans, these studies show that plasmid DNA targeted to dendritic cells can invoke an immune response without the use of an adjuvant. Thus our findings have important implications in the future of DNA immunizations in humans. These studies were done in a single strain of mouse; other strains need to be studied in order to determine whether this phenomenon is not species specific. Furthermore, different methods for introducing DNA need to be evaluated to determine which is the most efficient and least invasive for future use in humans.

\section{Acknowledgements}

The work was done in the laboratory of N.E.H and supported by the Novartis Research Foundation. We thank A. Lanzavecchia and J.S. Boyle for providing cell lines and plasmid encoding mouse CTLA4. We also thank other members of the N.E.H. laboratory for stimulating discussions. 


\section{References}

Aikawa, M., Hsieh, C.L. \& Miller, L.H. (1977) Ultrastructural changes of the erythrocyte membrane in ovale-type malarial parasites. Journal of Parasitology 63, 152-154.

Beerli, R.R., Wels, W. \& Hynes, N.E. (1994) Intracellular expression of single chain antibodies reverts ErbB-2 transformation. Journal of Biological Chemistry 269, 23931-23936.

Boyle, J.S., Barr, I.G. \& Lew, A.M. (1999) Strategies for improving responses to DNA vaccines. Molecular Medicine 5, 1-8.

Gardner, M.J., Doolan, D.L., Hedstrom, R.C., Wang, R., Sedegah, M., Gramzinski, R.A., Aguiar, J.C., Wang, H., Margalith, M., Hobart, P. \& Hoffman, S.L. (1996) DNA vaccines against malaria: immunogenicity and protection in a rodent model. Journal of Pharmaceutical Science 85, 1294-1300.

Hirunpetcharat, C., Tian, J.H., Kaslow, D.C., van Rooijen, N., Kumar, S., Berzofsky, J.A., Miller, L.H. \& Good, M.F. (1997) Complete protective immunity induced in mice by immunization with the 19kilodalton carboxyl-terminal fragment of the merozoite surface protein-1 (MSP1[19]) of Plasmodium yoelii expressed in Saccharomyces cerevisiae: correlation of protection with antigen-specific antibody titer, but not with effector CD4+ T cells. Journal of Immunology 159, 3400-3411.

Hoffman, S.L., Sedegah, M. \& Hedstrom, R.C. (1994) Protection against malaria by immunization with a Plasmodium yoelii circumsporozoite protein nucleic acid vaccine. Vaccine 12, 1529-1533.

McBride, J.S., Newbold, C.I. \& Anand, R. (1985) Polymorphism of a high molecular weight schizont antigen of the human malaria parasite Plasmodium falciparum. Journal of Experimental Medicine 161, 160-180.

Ockenhouse, C.F., Sun, P.F., Lanar, D.E., Wellde, B.T., Hall, B.T., Kester, K., Stoute, J.A.,
Magill, A., Krzych, U., Farley, L., Wirtz, R.A., Sadoff, J.C., Kaslow, D.C., Kumar, S., Church, L. W., Crutcher, J.M., Wizel, B., Hoffman, S., Lalvani, A., Hill, A.V., Tine, J.A., Guito, K.P., de Taisne, C., Anders, R., Ballou, W.R. \& et al. (1998) Phase I/IIa safety, immunogenicity, and efficacy trial of NYVAC-Pf7, a poxvectored, multiantigen, multistage vaccine candidate for Plasmodium falciparum malaria. Journal of Infectious Diseases 177, 1664-1673.

Sandhu, J.S. \& Kennedy, J.F. (1994) Expression of the merozoite surface protein gp195 in vaccinia virus. Vaccine 12, 56-64.

Sato, Y., Roman, M., Tighe, H., Lee, D., Corr, M., Nguyen, M.D., Silverman, G.J., Lotz, M., Carson, D.A. \& Raz, E. (1996) Immunostimulatory DNA sequences necessary for effective intradermal gene immunization. Science 273, 352-354.

Sedegah, M., Hedstrom, R., Hobart, P. \& Hoffman, S.L. (1994) Protection against malaria by immunization with plasmid DNA encoding circumsporozoite protein. Proceedings of the National Academy of Sciences 91, 98669870.

Soares, I.S., Levitus, G., Souza, J. M., Del Portillo, H.A. \& Rodrigues, M.M. (1997) Acquired immune responses to the $\mathrm{N}$ - and $\mathrm{C}$-terminal regions of Plasmodium vivax merozoite surface protein 1 in individuals exposed to malaria. Infection and Immunity 65, 16061614.

Tanabe, K., Mackay, M., Goman, M. \& Scaife, J.G. (1987) Allelic dimorphism in a surface antigen gene of the malaria parasite Plasmodium falciparum. Journal of Molecular Biology 195, 273-287.

Xiang, Z. \& Ertl, H.C. (1995) Manipulation of the immune response to a plasmid-encoded viral antigen by coinoculation with plasmids expressing cytokines. Immunity $\mathbf{2}$, 129-135. 